\title{
Sensibilité des chèvres Kirdimi et Sahéliennes du Tchad à la trypanosomose à Trypanosoma congolense
}

\author{
G. Ndoutamia ${ }^{1 *}$ A. Brahim ${ }^{1}$ P. Nadjindoroum ${ }^{1}$ \\ G. Moudaidandi ${ }^{1}$ G. Diimgang ${ }^{1}$ R. Loubadjim ${ }^{1}$
}

Mots-clés

Caprin - Chèvre Kirdimi - Chèvre Sahélienne - Trypanosoma congolense Infection expérimentale - Sang Biochimie - Réponse immunitaire Perte de poids - Tchad.

\begin{abstract}
Résumé
La trypanotolérance a fait l'objet d'une étude en station sur des chèvres du Tchad de races Sahélienne et Kirdimi. Cinquante-cinq (55) animaux, 28 Sahéliens et 27 Kirdimi, dont les types d'hémoglobine avaient été préalablement déterminés, ont été infectés expérimentalement, chacun avec $10^{6}$ trypanosomes de souche Trypanosoma congolense IL1180 (type savane). Les animaux ont été régulièrement suivis pendant six mois pour les signes cliniques, la croissance pondérale, les paramètres hématologiques et biochimiques. U ne perte significative et brutale de poids a été observée chez les chèvres Sahéliennes sous l'effet de cette infection. Chez les Kirdimi, en revanche, aucune variation significative n'a été remarquée. La période prépatente de 7 jours en moyenne chez les Sahéliennes est passée à 12 jours chez les Kirdimi. La parasitémie qui a semblé évoluer très rapidement chez les Sahéliennes, en l'espace d'un mois, a très bien été contrôlée par les Kirdimi pendant toute la durée de l'expérimentation et, dans certains cas, la disparition virtuelle des parasites a été observée. Les Sahéliennes, apparemment très vulnérables, ont eu, au plus fort de la maladie, de l'inappétence, une muqueuse oculaire pâle, un larmoiement, une démarche chancelante et quelquefois des diarrhées. L'hématocrite, stable chez les Kirdimi, a chuté rapidement et a souvent atteint le seuil critique de 15 p. 100 chez les Sahéliennes. Les animaux qui avaient atteint ce seuil étaient incapables de se relever et succombaient si un traitement trypanocide n'était pas appliqué. L'évolution de la trypanosomose à T. congolense s'est accompagnée, à des degrés différents, d'une modification considérable des paramètres hématologiques et biochimiques, surtout chez les chèvres Sahéliennes. Cette étude montre que les chèvres Kirdimi contrôlent mieux l'infection à T. congolense que les chèvres Sahéliennes.
\end{abstract}

\section{INTRO DUCTION}

La trypanosomose animale est une des contraintes majeures au développement de l'élevage en Afrique subsaharienne (5). L'infection est causée par des protozoaires flagellés du genre Trypanosoma. Elle se propage essentiellement par la piqûre d'insectes hématophages, notamment les glossines et les Tabanidés. La trypanosomose est répartie sur presque tout le territoire du Tchad. Elle affecte toutes les espèces animales et cause des pertes importantes (26). Diverses méthodes sont utilisées pour lutter contre cette

\footnotetext{
1. Laboratoire de recherches vétérinaires et zootechniques de Farcha, BP 433, N'Djaména, Tchad

Tél. : 527475 (ou 76) ; Fax : 527476

* Auteur pour la correspondance
}

maladie : la lutte anti-vectorielle, la chimiothérapie et l'utilisation du bétail trypanotolérant (17). La lutte antivectorielle est la méthode la plus classique. Elle a permis de réduire la densité des mouches tsé-tsé dans certaines régions de l'Afrique. Néanmoins, elle est très coûteuse et ne permet pas d'éradiquer la trypanosomose. La chimiothérapie est la méthode la plus répandue. Cependant, son utilisation anarchique favorise de plus en plus le développement de la chimiorésistance $(18,20)$. L'élevage de bétail trypanotolérant est un autre procédé de lutte considéré comme efficace contre la maladie (17).

Les informations disparates font état du caractère trypanotolérant de certaines races de chèvres et de moutons, mais on dispose de peu de renseignements précis sur leur degré de sensibilité (3). Ainsi, en Afrique de l'Ouest, les moutons et les chèvres de race Djallonké sont réputés trypanotolérants et leur habitat correspond 
aux zones infestées de glossines (3). De même, on remarque que les moutons et les chèvres Kirdimi vivent dans les zones subhumides infestées de glossines sans qu'apparemment leur productivité ne soit affectée. En revanche, les moutons et les chèvres de race Sahélienne seraient plus vulnérables. Ces observations ont amené à considérer les Kirdimi comme trypanotolérants (11). Comme aucune donnée expérimentale n'est disponible, cette étude a été entreprise pour vérifier cette présomption.

\section{MATERIEL ET METHODES}

Soixante (60) caprins, 30 Kirdimi et 30 Sahéliens, âgés, de 1 à 2 ans, ont été achetés respectivement aux alentours de Guelendeng et de Dourbali. Ces deux localités correspondent soit au berceau, soit à la zone d'extension de ces races de caprins.

Cinquante-cinq animaux ont été retenus sur la base du résultat négatif du test de détection des antigènes circulants de trypanosome (19). Deux groupes de caprins ont été constitués. Chaque groupe était constitué d'animaux de même race : 27 chèvres Kirdimi et 28 chèvres Sahéliennes.

\section{Traitement}

A leur arrivée au Laboratoire de Farcha, les animaux ont été traités avec un antibiotique (oxytétracycline, $20000 \mathrm{UI} / \mathrm{kg}$ ), un déparasitant interne (fenbendazole) et un trypanocide (acéturate de diminazène, $7 \mathrm{mg} / \mathrm{kg}$ ). Une mise en quarantaine d'un mois a été observée.

\section{Infection}

L'infection a été effectuée en inoculant par voie intraveineuse $10^{6}$ trypanosomes par animal. La souche de $T$. congolense était un clone dérivé de la souche de T. congolense IL1180 de l'Ilri (Nairobi) de type savane.

\section{Suivi des animaux}

Le suivi des animaux a consisté à observer régulièrement les signes cliniques et à déterminer les variations du poids, de l'hématocrite, des constantes hématologiques et biochimiques. Ce suivi a été quotidien pour les examens cliniques et la parasitémie, hebdomadaire pour l'hématocrite et les relevés de poids, mensuel pour la numération globulaire et les paramètres biochimiques.

\section{Mesure des poids}

Les animaux ont été pesés à l'aide d'un dynamomètre auquel ils avaient été suspendus par l'intermédiaire de sangles passées entre les pattes.

\section{Examen parasitologique}

La parasitémie a été estimée par l'examen microscopique de l'interphase des cellules sanguines et du plasma après centrifugation différentielle en tubes capillaires (16).

\section{Paramètres hématologiques et biochimiques}

Les prélèvements ont été effectués à la veine jugulaire avec des venojects dans des tubes sous vide avec et sans anticoagulant. Le sang recueilli dans les tubes avec anticoagulant a permis de faire la numération des globules rouges et blancs, tandis que celui qui a été recueilli dans le tube sec a permis d'obtenir du sérum pour les dosages biochimiques.

La numération globulaire a été faite en utilisant des unopettes (Beckton Deckinson). La dilution du sang a été préparée au 1/200 pour les globules rouges et au 1/40 pour les globules blancs. Les numérations des hématies et des leucocytes ont été effectuées à l'aide de la cellule hématimétrique de Malassez.

La formule leucocytaire a été établie après l'examen microscopique de l'étalement de sang sur lame colorée au May-GuinwaldGiemsa. Les analyses biochimiques ont été effectuées à l'aide d'un spectrophotomètre Spectronic 601 et les réactifs ont été de type Kit Biomérieux.

\section{Détermination des types d'hémoglobine des chèvres par électrophorèse}

Pour la préparation de l'hémolysat, $3 \mathrm{ml}$ de sang ont été recueillis dans un tube à essais et les globules ont été sédimentés et lavés trois fois avec du sérum physiologique. Au dernier lavage, le tube contenant la suspension des globules a été centrifugé à 5000 tours/min pendant cinq minutes. Une fois débarrassés du surnageant, les globules concentrés au fond du tube ont été dilués avec de l'eau distillée six fois leur volume et lysés ; puis $0,5 \mathrm{ml}$ de toluène par millilitre d'hémolysat a été ajouté, suivi d'un brassage au mixer pendant $30 \mathrm{sec}$. Le mélange a été à nouveau centrifugé pendant 10 min à 5000 tours/min pour que le déchet stromal se dépose. L'hémolysat a ensuite été transvasé dans un tube propre, bouché de façon étanche et conservé à $-20^{\circ} \mathrm{C}$ jusqu'à l'analyse.

L'électrophorèse de l'hémoglobine a été réalisée sur papier acétate de cellulose Phoroslide (Millipore). Le tampon de migration était à base de véronal sodique de $\mathrm{pH}=8,6$ et de force ionique 0,005 . La migration a été effectuée à une tension constante de 100 volts pendant $20 \mathrm{~min}$. La révélation des bandes qui représentaient les différents types d'hémoglobine a été faite en colorant le support en acétate de cellulose avec le ponceau S. Le support a ensuite été rincé trois fois dans 5 p. 100 de solution d'acide acétique, puis séché à température ambiante.

\section{Analyses statistiques}

Les comparaisons entre les groupes ont été faites en utilisant l'analyse de variance des écarts entre les moyennes par le logiciel Spss, en suivant la démarche d'Achukwi et coll. (1).

\section{RESU LTATS}

\section{Observations cliniques}

La pathologie de la trypanosomose des petits ruminants, bien que très peu documentée, est similaire à celle des autres espèces animales. Au plus fort de la maladie, les animaux ont de l'inappétence, présentent une muqueuse oculaire pâle, un larmoiement, ont une démarche chancelante et quelquefois des diarrhées. Ces signes cliniques n'ont pas été perceptibles chez la majorité des chèvres Kirdimi pendant toute la durée de l'expérimentation. Dix chèvres Sahéliennes et une chèvre Kirdimi sont mortes au cours de l'expérimentation. Les autopsies ont révélé des pétéchies. Les Sahéliennes ont présenté une baisse de poids régulière du début jusqu'à la fin de l'expérimentation (de 21,51 à 18,15 kg) (figure 1). Le poids des chèvres Kirdimi a semblé plutôt stable (figure 1). La période prépatente de 7 jours en moyenne chez les Sahéliennes est passée à 12 jours chez les Kirdimi. 


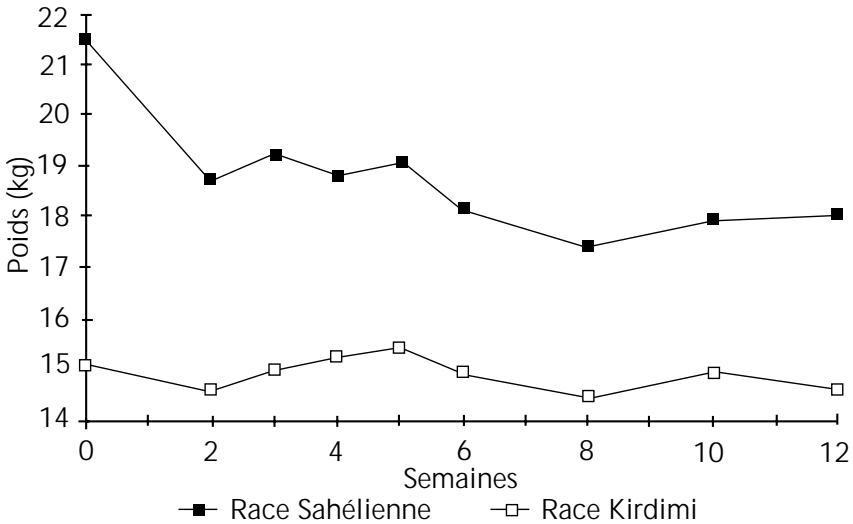

Figure 1 : variations du poids en fonction des races.

\section{Parasitémie}

Les parasitémies ont évolué en dents-de-scie. Elles sont apparues plus faibles pour les Kirdimi ou des cas de guérison spontanée ont été observés chez trois animaux. Chez les chèvres Sahéliennes, en revanche, les parasitémies ont évolué très rapidement. Elles ont varié de 20 à 200 trypanosomes par champ. Les animaux qui avaient atteint ce taux de parasitémie ont été incapables de se relever et ont subi un traitement au diminazène acéturate à la dose de $3,5 \mathrm{mg} / \mathrm{kg}$ de poids vif.

\section{Hématocrite}

L'évolution des hématocrites s'est composée d'une phase de déclin suivie d'une stabilité relative (figure 2). Chez les chèvres Sahéliennes, les hématocrites ont varié de 39 à 26 p. 100 et ont été stables pendant les deux premières semaines, puis ont chuté brutalement à partir de la deuxième semaine. Ils sont remontés lentement à la quatrième semaine pour se stabiliser aux alentours de 25 p. 100. Celui des chèvres Kirdimi a varié entre 27 et 24 p. 100. Ils ont été plus stables avec également une remontée à la quatrième semaine. Cette variation n'était pas significative. Un hématocrite de 15 p. 100 a été critique pour les Sahéliennes. Les animaux ayant atteint ce taux ont été incapables de se relever et ont succombé si un traitement au trypanocide n'était pas appliqué. Il a été nécessaire de fractionner la dose de trypanocide en deux et de les administrer séquentiellement dans un intervalle de 6 à 10 heures, sinon l'animal mourrait. En revanche, les chèvres Kirdimi ont facilement atteint le taux d'hématocrite de 15 p. 100 sans perturbation apparente. Il existe probablement un autre seuil critique de l'hématocrite caractéristique des Kirdimi à déterminer.

\section{Paramètres hématologiques}

\section{Numérations érythrocytaires et leucocytaires}

Chez les chèvres Sahéliennes, le nombre des globules rouges par microlitre a chuté de 6350000 à 3660000 en fin d'expérimentation (tableau I).

Les globules rouges des chèvres Kirdimi sont apparus plus stables. En effet, chez les Kirdimi, les globules rouges ont varié de

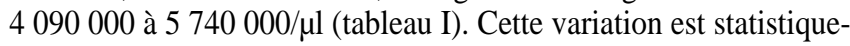
ment non significative $(\mathrm{P}>0,01)$.

Les variations des globules blancs au début et à la fin de l'expérimentation ne sont pas significatives chez les deux races de caprins.

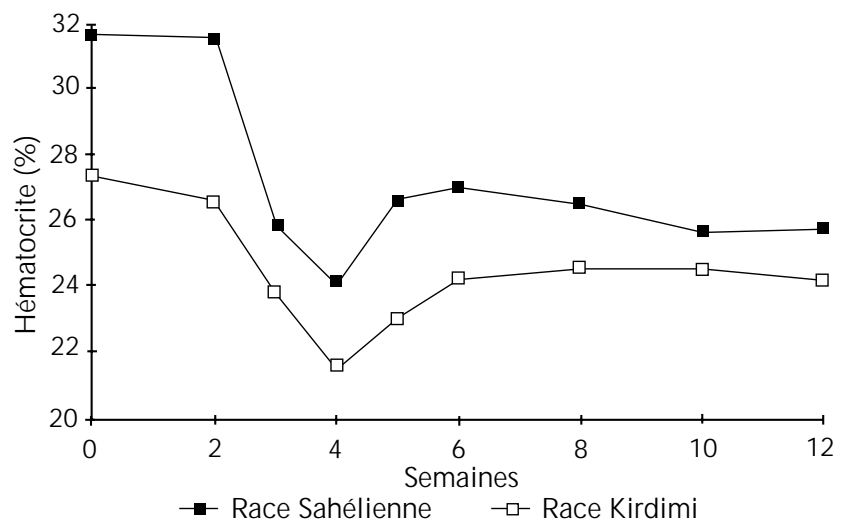

Figure 2 : variations de l'hématocrite en fonction des races.

Il est fort possible qu'une leucocytose passagère se soit produite, surtout durant la phase aiguë de la maladie. Néanmoins, on observe une redistribution de la formule leucocytaire à la fin de l'expérimentation (tableau I).

\section{Formule leucocytaire}

Le tableau I récapitule la formule leucocytaire avant et après l'infection. Chez les chèvres Kirdimi, les polynucléaires neutrophiles ont chuté de 43 à 31 p. 100 avec une augmentation concomitante des lymphocytes qui sont passés de 53 à 66 p. 100. Les taux de neutrophiles et de lymphocytes des chèvres Sahéliennes ont semblé stables pendant toute la durée de l'expérimentation. Chez tous les animaux, toutes races confondues, l'éosinophilie était très élevée et tendait à diminuer pour disparaître en fin d'expérimentation. Chez les Kirdimi, l'éosinophilie a varié de 2,4 à 0,7 p. 100 entre le début et la fin de l'expérimentation. Dans le groupe des Sahéliennes, elle a varié de 5 à 0,6 p. 100.

\section{Hémoglobine}

L'hémoglobine a chuté de 110 à 60 g/l chez les chèvres Sahéliennes. Chez les Kirdimi, la variation n'a pas été importante, elle a fluctué de 97 à $90 \mathrm{~g} / \mathrm{l}$.

\section{Bilirubines totales}

Les bilirubines ont varié de 4 à $39 \mathrm{mg} / \mathrm{l}$ chez les chèvres Sahéliennes. Elles ont été de 3 à $10 \mathrm{mg}$ chez les Kirdimi.

\section{Paramètres protéo-énergétiques}

Les paramètres protéo-énergétiques les plus caractéristiques relevés en début et en fin d'expérimentation sont consignés dans le tableau II. Les protéines sériques totales semblent ne pas avoir été affectées par la trypanosomose chez les animaux des deux races. Elles se sont stabilisées à $94 \mathrm{~g} / \mathrm{l}$ pour les Kirdimi et à $84 \mathrm{~g} / \mathrm{l}$ pour les Sahéliennes. Le taux d'albumine a chuté de 28 à $22 \mathrm{~g} / \mathrm{l}$ chez les chèvres Sahéliennes. Cette valeur a semblé stable chez les Kirdimi (30 et $33 \mathrm{~g} / \mathrm{l})$. L'urémie a diminué de 0,3 à $0,2 \mathrm{~g} / \mathrm{l}$ et a augmenté de 0,3 à $0,6 \mathrm{~g} / \mathrm{l}$ respectivement pour les Kirdimi et les Sahéliennes. La glycémie a chuté de 0,5 à $0,2 \mathrm{~g} / \mathrm{l}$ chez les chèvres Sahéliennes, en revanche, elle a très peu varié chez les Kirdimi $(0,5$ à $0,6 \mathrm{~g} / \mathrm{l})$. Le cholestérol a chuté de plus de la moitié de sa valeur initiale chez les animaux des deux races. Les lipides et les triglycérides n'ont pas varié chez les animaux des deux groupes. 


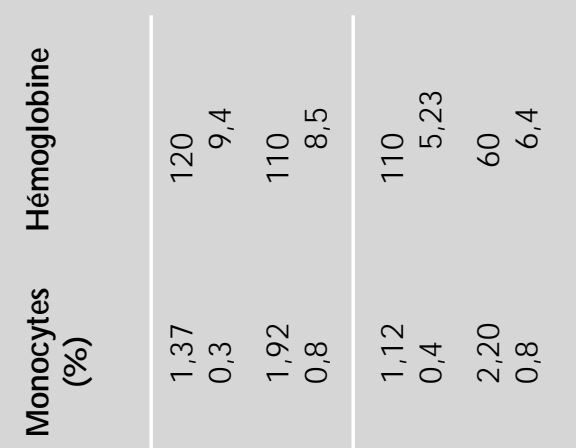

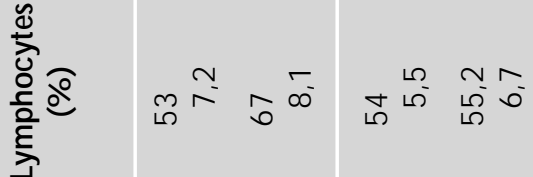

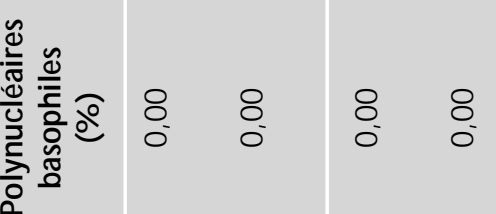

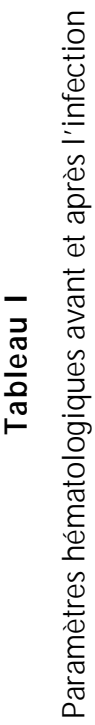

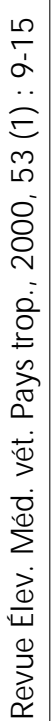

$\times 0 \times 0 \times 0 \times 0$

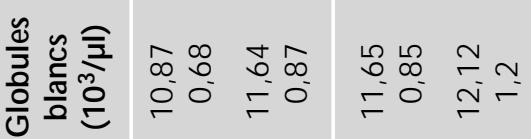

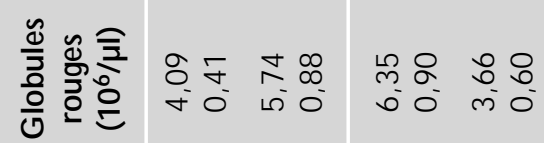

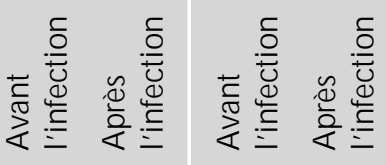

乎䒠

\&)

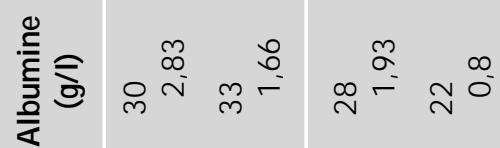

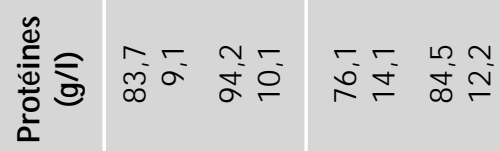

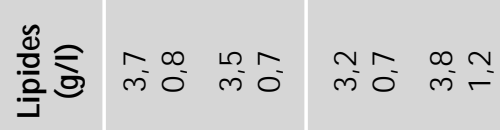

ए广

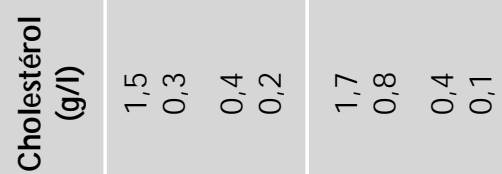

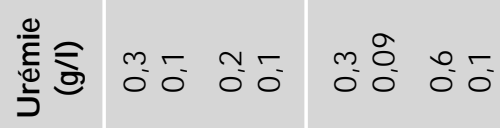

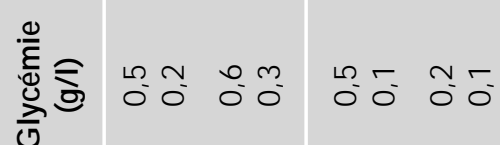
$\times 0 \times 0 \times 0 \times 0$

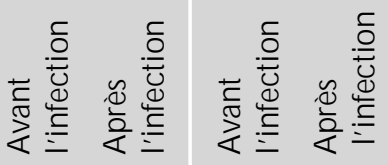

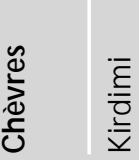




\section{Paramètres minéraux}

La concentration plasmatique du phosphore a varié considérablement chez les chèvres Kirdimi et Sahéliennes. Les valeurs de ce paramètre ont varié entre 62 et $112 \mathrm{mg} / \mathrm{l}$. L'infection n'a pas eu d'effet sur la teneur plasmatique du calcium et du magnésium chez les Kirdimi et chez les Sahéliennes. Les taux de calcium et de magnésium ont varié entre 77 et $110 \mathrm{mg} / \mathrm{l}$ chez les Kirdimi et entre 27 et $34 \mathrm{mg} / \mathrm{l}$ chez les chèvres Sahéliennes.

Le fer sérique a augmenté de 1,6 à 3,4 mg/l chez les chèvres Sahéliennes. En revanche, l'infection n'a pas paru influencer la valeur du fer sérique chez les Kirdimi (1,1 à 1,2 mg/l).

\section{Répartition des types d'hémoglobine}

Huit animaux étaient de type A, 17 de type B et 3 de type AB, chez les chèvres Sahéliennes. Chez les Kirdimi, un animal était de type A, 20 de type $B$ et 6 de type AB.

\section{Activité des enzymes}

L'activité de 5 ' nucléotidase a semblé stable chez les chèvres Sahéliennes et Kirdimi. Elle a varié de 3 à 4 U/l. L'activité de la lactate déshydrogénase a chuté de 495 à $90 \mathrm{U} / \mathrm{l}$ chez les chèvres des deux races. Chez les chèvres Sahéliennes, les transaminases ont augmenté de façon très significative. La transaminase glutamique oxalo-acétique (Tgo) a augmenté de 56 à 114 et la transaminase glutamo-pyruvique (Tgp) de 0,6 à 25 U/l. Chez les Kirdimi, les transaminases ont augmenté de 48,8 à 60,1 U/l et de 6 à 20 respectivement pour la Tgo et la Tgp. Cette variation n'est pas significative.

\section{DISCUSSION}

Les résultats de ce travail montrent des différences significatives de sensibilité à l'infection à $T$. congolense entre les chèvres de races Sahélienne et Kirdimi. Des facteurs innés ou acquis pourraient être à l'origine de cette différence de comportement (3). Compte tenu du manque de fiabilité du test utilisé pour la détection des antigènes circulants des trypanosomes, on ne doit pas écarter la possibilité que des animaux préalablement exposés à l'infection aient contribué en partie à cette différence de sensibilité chez les chèvres Sahéliennes et Kirdimi. Cette différence de sensibilité est reflétée à des degrés différents par l'anémie, la parasitémie, la croissance pondérale et les paramètres hématologiques et biochimiques. Les Kirdimi qui ont une période prépatente plus longue n'ont pas développé d'anémie perceptible. Elles ont mieux contrôlé la parasitémie que les Sahéliennes pendant toute la durée de l'expérience. En effet, ces observations viennent renforcer la tendance à considérer la trypanotolérance comme étant la capacité d'un animal à résister à l'anémie et à contrôler la trypanosomose $(13,17)$.

Paling and Dwinger (22) suggèrent même que les valeurs de l'hématocrite peuvent servir de critère unique de sélection pour la trypanotolérance. La chute de l'hématocrite a été faible chez les Kirdimi et brutale chez les Sahéliennes. Chez ces dernières, l'hématocrite s'est stabilisé à des valeurs basses à partir de la quatrième semaine. Ces observations concordent avec des résultats obtenus sur d'autres espèces $(10,23,25,27)$ qui indiquent que l'hématocrite décroît à l'apparition de la parasitémie. Il fluctue au cours de la maladie, avec même une tendance au rétablissement des valeurs normales chez les individus plus résistants. Quoique la parasitémie ait été évaluée par la méthode de score qui est une méthode semi-quantitative (12), il apparaît clairement que les Kirdimi contrôlent très bien la parasitémie.

Dans la présente expérience, la parasitémie de trois chèvres Kirdimi, estimée préalablement à plus de 20 trypanosomes par champ, a diminué progressivement pour devenir inapparente. Des efforts en vue de détecter les trypanosomes dans le sang périphérique de ces animaux se sont avérés vains ; du sang prélevé et inoculé à des souris suivies pendant trois mois n'a pas permis de détecter des trypanosomes, bien que cette souche soit très infectieuse chez les souris (20). On peut supposer que ces animaux ont été guéris spontanément (self-curing) (8). Il est à noter qu'il existait une différence de poids importante entre les animaux des deux races au début de l'expérience. Cette différence était due au petit format caractéristique des Kirdimi. Par conséquent, l'analyse de covariance a été mise à contribution pour prendre en compte cette différence de poids au départ (1). Il s'avère que les chèvres Kirdimi, bien que de petit format, ont perdu significativement moins de poids que les Sahéliennes dans les conditions d'expérimentation de cette étude.

L'infection à $T$. congolense a provoqué une chute significative du taux de l'hémoglobine, des érythrocytes et une augmentation du fer plasmatique et des bilirubines totales chez les Sahéliennes. En effet, la trypanosomose à $T$. congolense est une infection anémiante associée à une hémolyse intravasculaire (7). La baisse des érythrocytes et de l'hémoglobine serait la conséquence d'une destruction massive des globules rouges avec une augmentation concomitante des produits de métabolisme de l'hémoglobine, en l'occurrence les bilirubines. L'augmentation du fer plasmatique due à l'infection à $T$. brucei et à $T$. congolense a déjà été observée par Ogunsanmi et coll. (21). Cette augmentation pourrait être attribuée à une défaillance dans la mobilisation du fer, due à un dysfonctionnement de la moelle osseuse (29) et de l'hématopoï̀̀se.

Les changements intervenus chez les chèvres des deux races au niveau des formules leucocytaires peuvent être considérés comme des réactions de défense des animaux. En effet, l'augmentation des lymphocytes peut être associée à une hausse des globulines, des IgM en particulier et, dans une moindre mesure, des IgG (7). Quant à la diminution des éosinophiles, elle est couramment rapportée lors d'infections à Trypanosoma (2). Il est fort possible que le stress provoqué par l'infection à $T$. congolense ait induit une sécrétion accrue de l'adrénaline, connue pour son effet suppresseur de l'éosinophilie (30).

Les animaux infectés de race Sahélienne ont développé une hypoalbuminémie et une hypoglycémie. Les variations au niveau des protéines plasmatiques n'ont pas été significatives chez les animaux des deux races. Ces résultats ont déjà été rapportés lors d'une trypanosomose expérimentale chez des ovins $(14,21)$.

Il semble que les parasites, plus particulièrement les trypanosomes qui envahissent le système sanguin, accroissent les besoins de l'animal en protéines et en glucose, car ces substances constituent le principal support utilisé dans le métabolisme des parasites (15). C'est ce qui expliquerait la chute du taux d'albumine et de glucose chez les Sahéliennes. L'augmentation de l'urée sanguine observée chez les chèvres Sahéliennes dans cette expérimentation concorde avec les résultats d'Ogunsanmi et coll. (21) pour les infections à T. brucei. Cette augmentation considérable de l'urée sanguine est probablement due au dysfonctionnement des reins (21). En revanche, la faible fluctuation de ce paramètre sanguin chez les Kirdimi pourrait s'expliquer par la résistance des animaux à la rupture de l'équilibre physiologique des reins, sous la pression de l'infection à Trypanosoma congolense. 
Les variations du phosphore, du calcium et du magnésium ne concordent pas avec les résultats d'Ogunsanmi et coll. (21) qui observent pour les ovins infectés avec T. brucei une augmentation du calcium et une baisse de phosphore. Il est possible que les différentes espèces de trypanosomes et d'animaux utilisés dans les expérimentations soient à l'origine de la divergence des résultats (12).

Les activités des transaminases, en l'occurrence la Tgo et la Tgp, ont augmenté pendant toute la durée de l'infection chez les chèvres Sahéliennes. Les transaminases sont des enzymes qui ont une localisation spécifique dans des organes, notamment le foie. La hausse de leur activité dans le sérum pouvait témoigner d'une atteinte du tissu hépatique.

La détermination de la nature de l'hémoglobine des Sahéliennes et des Kirdimi de cette étude a montré que l'hémoglobine A n'était pas très caractéristique de ces animaux. En revanche, la proportion d'hémoglobine B a été relativement élevée. Le N'Dama, taurin reconnu comme étant trypanotolérant (6), possède 100 p. 100 d'hémoglobine A ; ce type d'hémoglobine serait associée à cette trypanotolérance (24). Si c'est le cas, le polymorphisme de l'hémoglobine (sous réserve de confirmation par un échantillon plus représentatif) ne serait pas lié au bon comportement des Kirdimi de cette étude face à l'infection à $T$. congolense. Des recherches orientées vers d'autres protéines pourraient permettre de déterminer les marqueurs responsables de cette trypanotolérance. En effet, la trypanotolérance est un caractère avec des causes mulifactorielles d'ordre génétique, se traduisant par un comportement immunologique particulier, sous la dépendance des facteurs écologiques $(9,28)$. L'identification des marqueurs génétiques par la mise à contribution de la biotechnologie pourrait permettre de mieux caractériser la trypanotolérance. La découverte de tels marqueurs apporterait un nouvel élan à la lutte contre la trypanosomose car elle permettrait de sélectionner les animaux résistants, même en l'absence de la maladie.

Les chèvres Kirdimi sont trypanotolérantes, probablement à la suite d'un long processus d'adaptation aux infections répétées dues à la trypanosomose. En revanche, les chèvres Sahéliennes ont sans doute une histoire qui n'est pas très liée à la trypanosomose. La trypanotolérance est un caractère héréditaire et quantitatif (21) et, en tant que tel, le brassage entre les deux races peut entraîner une dilution de ce caractère (4). La mobilité des troupeaux, très ancrée dans la tradition tchadienne, concerne 75 p. 100 des éleveurs. Elle permet, avec de faibles coûts, une production extensive, mais très adaptée aux difficiles conditions climatiques et socioculturelles du milieu sahélien. Cependant, cette pratique crée des conditions de métissage favorables entre les chèvres Sahéliennes et Kirdimi. En effet, dans les zones de transition des deux races (entre le $10^{\mathrm{e}}$ et le $11^{\mathrm{e}}$ parallèle) on observe un fort métissage des deux races. Il serait alors opportun de réguler ce croisement dans l'esprit de concilier le caractère résistant des Kirdimi et les caractéristiques de production (grand format, production laitière...) comparativement avantageuses des Sahéliennes.

\section{- CONCLUSION}

Cette étude a montré que les chèvres Kirdimi sont trypanotolérantes alors que les chèvres Sahéliennes sont trypanosensibles. Les Kirdimi devraient en principe survivre sans trop de problème dans les zones où la trypanosomose sévit à l'état endémique. L'exploitation des chèvres Kirdimi pourrait constituer une stratégie durable, sans effet néfaste pour l'environnement, dans le contrôle de la trypanosomose au Tchad.

\section{Remerciements}

Cette étude a été réalisée grâce à l'appui financier du Fonds d'aide et de coopération, dans le cadre du projet d'appui au secteur de l'élevage du Tchad oriental (Aseto), composante II (projet/ CD/94). L'exécution de ce projet n'aurait pu être possible sans la collaboration du personnel du Laboratoire de recherches vétérinaires et zootechniques de Farcha auquel nous exprimons notre profonde gratitude.

\section{BIBLIO GRAPHIE}

1. ACHUKWI M.D., TANYA V.N., HILL E.W., BRADLEY D.G., MEGHEN C., SAUVERO CHE B., BANSER J.T., NDO KI J.N., 1997. Susceptibility of the Namchi and Kapsiki cattle of Cameroon to trypanosome infection. Trop. Anim. Health Prod., 4: 219-226.

2. ANOSA V.O., 1988. Haematological and biochemical changes in human and animal trypanosomiasis. Part I. Revue Elev. M éd. vét. Pays trop., 41: 65-78.

3. BENGALY Z., CLAUSEN P.H., BOLY H., KANWE A., DUVALET G., 1993. Comparaison de la trypanosomose expérimentale chez certaines races de petits ruminants au Burkina Faso. Revue Elev. Méd. vét. Pays trop., $46:$ 563-570.

4. BRADLEY D.G., MACHUGH D.E., LOFFUS R.T., SOW R.S., HOSTE C.H., CUNNINGHAM E.P., 1994. Zebu taurine variation in $Y$ chromosomal DNA; a sensitive assay for genetic introgression in West African trypanotolerant cattle populations. Anim. Genet., 25: 7-12.

5. BRADY J., 1991. Seeing flies from space. Nature, 351: 695.

6. CHANDLER R.L., 1958. Studies on tolerance of $\mathrm{N}$ dama cattle to trypanosomiasis. J. comp. Path. Ther., 68: 253-260.

7. DARGIE J.D., MURRAY P.K., GRINSHAW W.T.R., M CINTYRE W.I.M., 1979. Bovine trypanosomiasis: the red cells kinetics of N dama and Zebu cattle infected with Trypanosoma congolense. Parasitology, 78: 271-286.

8. DIA M.L., AMINETOU M., DIOP C.,THIAM A., 1997. Auto-guérison chez un chamelon (Camelus dromedarius) expérimentalement infecté par Trypanosoma evansi. Revue Méd. vét., 148 : 713-716.

9. D'IETEREN G.D.M., AUTHIE E., WISSOCQ N., MURRAY M., 1998. Trypanotolerance, an option for sustainable livestock production in areas at risk from trypanosomosis. Revue sci. tech. O ff int. Epizoot., 17: 154-175.

10. DOKO A., VERHULST A., PANDEY V.S., VAN DER STUYFT P., 1997. Trypanosomose expérimentale à Trypanosoma brucei brucei chez les taurins Holstein et les zébus Bororo blancs. Revue Elev. Méd. vét. Pays trop., 50 : 23-28.

11. DUMAS R.V., 1977. Etude sur l'élevage des petits ruminants au Tchad. M aisons-Alfort, France, G erdat-lemvt, 355 p. (Rapport $n^{\circ} 15$ )

12. HERBERT W.J., LUMSDEN W.H.R., 1976. Trypanosoma brucei: a rapid method for estimating the host's parasitaemia. Exp. Parasitol., 49: 427-431.

13. ILCA, 1992. Trypanotolerant Livestock in West and Central Africa, Vol 3. A decade's results. Addis Abeba, Ethiopia, International Livestock Centre for Africa (ILCA), p. 206. (M onograph N o. 2)

14. KATUNGUKA-RWAKISHAYA E., 1996. Influence of Trypanosoma congolense infection on some blood inorganic and protein constituents in sheep. Revue Elev. M éd. vét. Pays trop., 49: 311-314.

15. LENG R.A., 1981. Modification of rumen fermentation. In: Proc. International Symposium on Nutritional Limits to Animal Production from Pastures, St Lucia, Q ueensland, Australia, 24-28 August 1981. Farnham Royal, UK, JcB Hacker, p. 427-453.

16. MURRAY M., MURRAY P.K., M CINTYRE W.I.M., 1977. Improved parasitological technique for the diagnosis of African trypanosomiasis. Trans. R. Soc. trop. Med. Hyg., 71: 325-326.

17. MURRAY M., STEAR M.J., TRAIL J.C.M., D'IETEREN G.D.M., AGYEMANG K., DWINGER R.H., 1991. Trypanosomiasis in cattle prospects for control. In: O wen J.B., Axford R.F.C. Eds., Breeding for disease resistance in farm animals. Farnham Royal, UK, CAB International, p. 203-234. 
18. MWAMBU P.M., MAYENDE J.S.P., 1971. Occurrence of berenil resistant strains of T. vivax. Trans. R. Soc. trop. Med. Hyg., 65: 254-255.

19. NANTULYA V.M., LINDQVIST K.I., 1989 Antigen detection enzyme immunoassays for diagnosis of Trypanosoma vivax, T. congolense and T. brucei infections in cattle. Trop. Med. Parasitol., 40: 267-272.

20. NDOUTAMIA G., MOLOO S.K., MURPHY N.B., PEREGRINE A.S., 1993. Derivation and characterisation of a quinapyramine resistant clone of Trypanosoma congolense. Antimicrob. Agents Chemother., 37: 1163-1166.

21. OGUNSANMI A.O., AKPAVIE S.O., ANOSA V.O., 1994. Serum biochemical changes in West African Dwarf sheep experimentally infected with Trypanosoma brucei. Revue Elev. Méd. vét. Pays trop., 47: 195-200.

22. PALING R.W., DWINGER R.H., 1993. Potential of trypanotolerance as a contribution to sustainable livestock production in tsetse affected areas in Africa. Vet. Q., 15: 60-67.

23. PALING R.W., MOLOO S.K., SCOTT J.R., MCODIMBA F.A., LOGAN-HENFREY L.I., MURRAY M., WILLIAM D.J.L., 1991. Susceptibility of Ndama and Boran cattle to tsetse transmitted primary and challenge infections with an homologous serodeme of Trypanosoma congolense. Parasite Immunol., 13: 413-425.

\section{Summary}

N doutamia G., Brahim A., Nadjindoroum P., Moudaidandi G., Diimgang G., Loubadjim R. Sensitivity of Kirdimi and Sahelian goats of Chad to Trypanosoma congolense infection

Sahelian and Kirdimi goats of Chad were studied in an experimental farm for trypanotolerance. Fifty-five (55) animals, 28 Sahelians and 27 Kirdimis, whose hemoglobin types had been determined, were experimentally infected, each one with $10^{6}$ trypanosomes (Trypanosoma congolense IL1180 stock, savanna type). The animals were regularly monitored for six months for clinical symptoms, bodyweight, hematological and biochemical parameters. The infection induced sudden significant weight loss in Sahelian goats. On the other hand, no significant weight variations were observed in Kirdimis. The average prepatent period was 7 and 12 days for Sahelian and Kirdimi goats, respectively. Parasitemia appeared to develop quickly in Sahelian goats (within a month). It was well under control in Kirdimis throughout the experimental period, and some self-cure cases were even observed. Sahelian goats, apparently more susceptible to the infection, displayed during the acute phase of the disease lack of appetite, pale ocular membranes, watering eyes, staggering movements and occasional diarrheas. The packed cell volume was stable in Kirdimis. It dropped rapidly in Sahelian goats and often reached the critical point of $15 \%$. At that threshold the animals were unable to stand up and died unless a trypanocide treatment was applied. The $T$. congolense trypanosomosis evolution was mainly associated in Sahelian goats and at different levels with important changes in hematological and biochemical parameters. This study shows that Kirdimi goats control T. congolense infections better than Sahelian goats.

Key words: Kirdimi goat - Sahelian goat - Trypanosoma congolense - Experimental infection - Blood - Biochemistry Immune response - W eight loss - Chad.
24. PETIT J.P., 1968. Détermination de la nature des hémoglobines chez 982 bovins africains et malgaches (taurins et zébus) par éléctrophorèse sur acétate de cellulose. Revue. Elev. Méd. vét. Pays trop., 21 : 405-413.

25. PINDER M., LIBEAU G., HIRSCH G., TAMBOURA I., HAUCKBAUER R., ROELANTS C.E., 1984. Anti-trypanosome specific immune responses in bovids of differing susceptibility to African trypanosomiasis. Immunology, 51: 337-341.

26. RECEVEUR P., 1938. Notes sur certaines affections du cheptel des régions Nord-Est du Tchad. Recl Méd. vét. exot., 10 : 113-118.

27. ROELANTS C.E., TAMBO URA I., SIDIKI D.B., BASINGA A., PINDER M., 1983. Trypanotolerance and individual note a breed character. Acta trop., 40: 99-104

28. TOURE S.M., 1977. La trypanotolérance. Revue des connaissances. Revue. Elev. M éd. vét. Pays trop., $30:$ 157-174.

29. WELLDE B.T., LOTZSCH R., DEINOL G., SANDUN E., WILLIAMS J., WARUT G., 1974. Trypanosoma congolense. Clinical observations of experimentally infected cattle. Exp. Parasitol., 36: 6-19.

30. WINTRO BE M.M., 1974. Clinical haematology. 7th ed. Philadelphia, PA, USA, Lea and Febinger, p. 215-227.

Reçu le 26.04.99, accepté le 18.10 .00

\section{Resumen}

N doutamia G., Brahim A., Nadjindoroum P., Moudaidandi G., Diimgang G., Loubadjim R. Sensibilidad de las cabras Kirdimi y Saharianas de Chad a la tripanosomosis por Trypanosoma congolense

La tolerancia al tripanosoma (tripanotolerancia) fue el objeto de un estudio en la estación, llevado a cabo con cabras de Chad de las razas Sahariana y Kirdimi. Cincuenta y cinco (55) animales, 28 Saharianos y 27 Kirdimi, cuyos tipos de hemoglobina habían sido determinados con anterioridad, fueron infectados en forma experimental, cada uno con 106 tripanosomas de la cepa Trypanosoma congolense IL1180 (tipo sabana). Los signos clínicos, el crecimiento ponderal y los parámetros hematológicos y bioquímicos de los animales fueron seguidos regularmente durante seis meses. En las cabras Saharianas bajo el efecto de esta infección, se observó una pérdida significativa y brutal del peso. En las Kirdimi, por el contrario, no se observó ninguna variación significativa. El periodo de latencia en promedio de 7 días en las Saharianas pasó a 12 días en las Kirdimi. La parasitemia, con una evolución aparentemente muy rápida en las Saharianas, de un mes, fue muy bien controlada por las Kirdimi durante toda la duración del experimento $y$, en ciertos casos, se observó una desaparición virtual de los parásitos. Las Saharianas, aparentemente vulnerables, presentaron, en el pico de la enfermedad, falta de apetito, mucosas oculares pálidas, lagrimeo, caminar vacilante y algunas veces diarrea. El hematocrito, estable en las Kirdimi, cayó rápidamente y a menudo alcanzo un nivel crítico de $15 \%$ en las Saharianas. Los animales que alcanzaban este nivel eran incapaces de levantarse y hubiesen perecido si no se hubiese administrado un tratamiento tripanocida. La evolución de la tripanosomosis por T. congolense se acompaña, a niveles variables, de una modificación considerable de los parámetros hematológicos y bioquímicos, sobre todo en las cabras Saharianas. Este estudio demuestra que las cabras Kirdimi controlan mejor la infección por T. congolense que las cabras Saharianas.

Palabras clave: Caprino - Cabra Kirdimi - Cabra Saheliana Trypanosoma congolense - Infección experimental - Sangre Bioquímica - Perdida de peso - Chad. 